\title{
Complete maximal surfaces in static Robertson-Walker 3-spaces
}

\author{
Isabel Fernández • Pablo Mira
}

\begin{abstract}
We construct a large family of complete maximal surfaces in the static Robertson-Walker 3-spaces with negative base curvature. These examples provide a wide variety of non-standard foliations of such spaces by entire maximal graphs.
\end{abstract}

\section{Introduction}

A maximal hypersurface in a Lorentzian manifold $\mathcal{M}^{n+1}$ is a spacelike hypersurface of $\mathcal{M}^{n+1}$ whose mean curvature vanishes at every point. Apart from their obvious geometrical importance, maximal hypersurfaces play a relevant role in different problems in General Relativity (see for instance [11] and references therein).

The fundamental result on the global behaviour of maximal hypersurfaces is the Calabi-Bernstein theorem [6,7], which states that the only complete maximal hypersurfaces in the Minkowski space are the spacelike hyperplanes. This result has inspired many other works that seek extensions of the Calabi-Bernstein theorem to more general Lorentzian manifolds (see $[1-3,10]$ for instance). In particular, special attention has been paid to the case in which the ambient manifold is a Generalized Robertson-Walker (GRW for short) space. Thus, the Calabi-Bernstein problem in these spaces asks if any complete maximal hypersurface in a GRW space is necessarily a spacelike slice.

\footnotetext{
I. Fernández ( $)$ )

Departamento de Matematica Aplicada I, Universidad de Sevilla, 41012 Sevilla, Spain e-mail: isafer@us.es

P. Mira

Departamento de Matemática Aplicada y Estadística, Universidad Politécnica de Cartagena, 30203 Cartagena, Murcia, Spain

e-mail: pablo.mira@upct.es
} 
The two-dimensional version of this problem has received a special attention. For instance, in $[1,10]$ it is proved that the Calabi-Bernstein theorem holds for a large class of GRW 3-spaces. Nonetheless, this situation changes in many other GRW spaces. For instance, in the case of a static Robertson-Walker (RW) 3-space $M^{2}(\kappa) \times \mathbb{R}$ with constant negative base curvature $\kappa<0$ and product metric $\langle,\rangle_{M}-d t^{2}$, some particular complete maximal surfaces that are not slices were found in [1].

Our purpose in this note is to construct an extremely large family of complete maximal surfaces in the static RW 3-spaces $M^{2}(\kappa) \times \mathbb{R}, \kappa<0$. More specifically, we will associate to each holomorphic 1-form $\omega$ on the unit disk $\mathbb{D}$ that is bounded with respect to the Poincaré norm a different example of a complete maximal surface. This result illustrates a very rich global structure, and seems to be the first general construction procedure of complete maximal surfaces other than slices on a RW space. The proof relies on a previous theorem by the authors in [9].

Finally, it is remarkable that the examples constructed here provide a large family of non-standard foliations of the static RW 3-spaces by entire maximal graphs. This property might be relevant since the Einstein equations are quite simplified when viewed as the time evolution of an initial maximal graph. An explanation of this fact is given in [4] (see also [8]).

\section{Preliminaries}

A generalized Robertson-Walker space (GRW space) is a product manifold $M^{n} \times I$ of a complete Riemannian manifold $\left(M^{n}, g\right)$ with an interval $I \subset \mathbb{R}$, which is endowed with the Lorentzian warped product metric

$$
\langle,\rangle=-\pi_{I}^{*}\left(d t^{2}\right)+f\left(\pi_{I}\right)^{2} \pi_{M}^{*}(g) .
$$

Here $\pi_{I}, \pi_{M}$ are the projections onto the base $M^{n}$ and the fiber $I$, and $f: I \rightarrow$ $(0,+\infty)$ is a smooth function. When $M^{n}$ has constant curvature, $M^{n} \times I$ is classically known as a Robertson-Walker space. It is complete if $I=\mathbb{R}$, and static if $f$ is constant.

A hypersurface $\Sigma^{n} \subset M^{n} \times I$ is said to be spacelike if the metric induced on $\Sigma^{n}$ from the ambient metric (2.1) is a Riemannian metric. It is called a maximal hypersurface if, additionally, its mean curvature $H$ vanishes identically. Let us remark the following properties of spacelike hypersurfaces in a GRW space $[2,3,10]$.

(i) The simplest hypersurfaces are the slices $M^{n} \times\left\{t_{0}\right\}$. These slices always have constant mean curvature, and are maximal hypersurfaces exactly when $f^{\prime}\left(t_{0}\right)=0$.

(ii) Any spacelike hypersurface that is complete (i.e. its induced metric is complete in the usual sense) is an entire graph over the base $M^{n}$. In contrast, not every entire graph on a GRW space has a complete induced metric.

(iii) If $\eta$ denotes the unit normal of a spacelike surface in the GRW space $M^{2} \times I$, and $t$ denotes the coordinate of the factor $I$, then it holds (see Eq. (9) in [10])

$$
\Delta t=-\frac{f^{\prime}(t)}{f(t)}\left\{2+\|\nabla t\|^{2}\right\}+2 H\left\langle\eta, \partial_{t}\right\rangle .
$$


Here $\Delta, \nabla,\|\cdot\|$ denote, respectively, the Laplacian, the gradient and the norm on the surface with respect to its induced metric.

Let now $M^{2}(\kappa)$ denote the two-dimensional Riemannian space form of constant curvature $\kappa<0$, and let us view it as a hyperboloid of Minkowski 3-space $\mathbb{L}^{3}$ in the usual way, i.e.

$$
M^{2}(\kappa)=\left\{\left(x_{0}, x_{1}, x_{2}\right) \in \mathbb{L}^{3}:-x_{0}^{2}+x_{1}^{2}+x_{2}^{2}=\frac{-1}{\sqrt{-\kappa}}, x_{0}>0\right\} .
$$

In addition, let us recall the Wirtinger operators associated to a complex parameter $z=s+i t$ of a complex domain $\Omega \subset \mathbb{C}$ :

$$
\frac{\partial}{\partial z}:=\frac{1}{2}\left(\frac{\partial}{\partial s}-i \frac{\partial}{\partial t}\right), \quad \frac{\partial}{\partial \bar{z}}:=\frac{1}{2}\left(\frac{\partial}{\partial s}+i \frac{\partial}{\partial t}\right) .
$$

Then we have the following notion:

Definition 2.1 A map $N: \Omega \subset \mathbb{C} \rightarrow M^{2}(\kappa) \subset \mathbb{L}^{3}$ is harmonic if $N_{z \bar{z}}$ is everywhere orthogonal to $T_{N} M^{2}(\kappa)$.

Equivalently, $N$ is harmonic iff $N_{z \bar{z}}$ is collinear with $N$, iff $Q:=\left\langle N_{z}, N_{z}\right\rangle d z^{2}$ is a holomorphic quadratic differential on $\Omega \subset \mathbb{C}$. For further details on harmonic maps into $M^{2}(\kappa)$ we refer to [9].

Finally, let us recall that $M^{2}(-1)$ may be viewed via stereographic projection in $\mathbb{L}^{3}$ as the unit disk $\mathbb{D}$ endowed with the Poincaré metric

$$
d s_{P}^{2}:=\frac{4|d z|^{2}}{\left(1-|z|^{2}\right)^{2}}
$$

for each $z \in \mathbb{D}$.

\section{The main result}

Theorem 3.1 Let $\omega$ denote a holomorphic 1-form on the unit disk $\mathbb{D}$ that is bounded in the Poincaré norm of $\mathbb{D}$, i.e.

$$
\|\omega\|^{2}:=\sup _{\mathbb{D}} \frac{|\omega|^{2}}{d s_{P}^{2}}<+\infty
$$

Then there exists a harmonic diffeomorphism $N: \mathbb{D} \rightarrow M^{2}(\kappa)$ associated to $\omega$ such that

$$
f^{\omega}:=\left(N, 2 \operatorname{Re} \int \omega\right): \mathbb{D} \rightarrow M^{2}(\kappa) \times \mathbb{R}
$$

is a complete maximal surface in the static Robertson-Walker space $M^{2}(\kappa) \times \mathbb{R}$. Moreover, if $\omega_{1} \neq \pm \omega_{2}$ the surfaces $f^{\omega_{1}}, f^{\omega_{2}}$ are non-congruent. 
Proof It is obvious that, up to dilations, we may assume that $\kappa=-1$ (and thus $M^{2}(\kappa)=\mathbb{H}^{2}$, the canonical hyperbolic plane), and that $f=1$ since $f$ is constant.

Let $\omega$ denote a holomorphic 1 -form on $\mathbb{D}$. Then by [9, Theorem 22] there exists a harmonic map $N: \mathbb{D} \rightarrow \mathbb{H}^{2}$ such that:

a) $\langle d N, d N\rangle=\omega^{2}+\mu|d z|^{2}+\bar{\omega}^{2}$, where $\mu$ is a non-negative smooth function.

b) $N$ is a global diffeomorphism onto its image $N(\mathbb{D})$. In particular, $d N$ is everywhere regular, and so $\mu|d z|^{2}>2|\omega|^{2}$ holds.

c) The metric $\lambda^{*}|d z|^{2}:=\mu|d z|^{2}+2|\omega|^{2}$ is complete.

Moreover, the harmonic map $N$ is unique with these conditions (up to isometries of $\mathbb{H}^{2}$ ), and if $N_{1}, N_{2}$ are induced by two different holomorphic 1-forms $\omega_{1} \neq \pm \omega_{2}$, then $N_{1}$ and $N_{2}$ are non-congruent in $\mathbb{H}^{2}$ [9, Corollary 23].

If we now look at (3.2), and denote the last coordinate by $t$, it follows that $t_{z} d z=\omega$. Thereby, $\left\langle f_{z}^{\omega}, f_{z}^{\omega}\right\rangle=0$ and the induced metric on $\mathbb{D}$ by the pullback via $f^{\omega}$ of the ambient Lorentzian metric on $M^{2}(-1) \times \mathbb{R}$ is

$$
\left\langle d f^{\omega}, d f^{\omega}\right\rangle=\mu|d z|^{2}-2|\omega|^{2}=\lambda^{*}|d z|^{2}-4|\omega|^{2} .
$$

As $\mu|d z|^{2}>2|\omega|^{2}$, we see that $\lambda|d z|^{2}:=\mu|d z|^{2}-2|\omega|^{2}$ is a Riemannian metric, i.e. $f^{\omega}$ is a spacelike surface in the Robertson-Walker space $M^{2}(-1) \times \mathbb{R}$. Moreover, $\Delta t=(4 / \lambda) t_{z \bar{z}}=0$ as $t$ is harmonic on $\mathbb{D}$. Besides, since $N$ is a local diffeomorphism the unit normal of $f^{\omega}$ is nowhere horizontal. So by (2.2) we can conclude that $f^{\omega}$ has vanishing mean curvature, i.e. it is a maximal surface.

It remains to check that the metric (3.3) is complete. First, let us recall that $\omega$ is bounded in the Poincaré norm. Then [12, Theorem 13] ensures that the harmonic map $N: \mathbb{D} \rightarrow \mathbb{H}^{2}$ is quasiconformal. Hence, the dilation coefficient $D_{N}$ of $N$ (see [5]) is bounded. Let us now compute $D_{N}$. Taking into account the metric expression of $\langle d N, d N\rangle$ exposed in a), it is a simple computation to check that the eigenvalues $\rho_{1} \geq \rho_{2}$ of the metric $\langle d N, d N\rangle$ are

$$
\rho_{1}|d z|^{2}=\frac{1}{2} \mu|d z|^{2}+|\omega|^{2}, \quad \rho_{2}|d z|^{2}=\frac{1}{2} \mu|d z|^{2}-|\omega|^{2} .
$$

Therefore (see [5])

$$
D_{N}^{2}=\frac{\rho_{1}}{\rho_{2}}=\frac{\mu|d z|^{2}+2|\omega|^{2}}{\mu|d z|^{2}-2|\omega|^{2}}=\frac{\lambda^{*}}{\lambda}
$$

Thus, as $D_{N}$ is bounded, there is some positive constant $C$ such that $\lambda^{*} \leq C \lambda$. As $\lambda^{*}|d z|^{2}$ is a complete metric, so is $\lambda|d z|^{2}$.

Finally, as we said previously, if $\omega_{1} \neq \pm \omega_{2}$, by [9, Corollary 23] the harmonic maps $N_{1}, N_{2}$ respectively associated to $\omega_{1}, \omega_{2}$ are non-congruent in $\mathbb{H}^{2}$. Thus $f^{\omega_{1}}$ cannot be congruent to $f^{\omega_{2}}$. This ends up the proof.

Remark 3.2 All the examples given by Theorem 3.1 are entire graphs over the base $M^{2}(\kappa)$ of the static Robertson-Walker space $M^{2}(\kappa) \times \mathbb{R}$, since they are complete. Moreover, as translations along the fibers are isometries, we conclude that any of 
the complete maximal surfaces in Theorem 3.1 provides a non-standard foliation of $M^{2}(\kappa) \times \mathbb{R}$ by entire maximal graphs.

Acknowledgments I. Fernández was partially supported by MEC-FEDER, grant no. MTM 2004-00160. P. Mira was partially supported by MEC-FEDER, grant no. MTM 2004-02746.

\section{References}

1. Albujer, A., Alías, L.J.: Calabi-Bernstein results for maximal surfaces in Lorentzian product spaces. (2006) (preprint)

2. Alías, L.J., Romero, A., Sánchez, M.: Spacelike hypersurfaces of constant mean curvature in certain spacetimes. Nonlinear Anal. 30, 655-661 (1997)

3. Alías, L.J., Romero, A., Sánchez, M.: Uniqueness of complete spacelike hypersurfaces of constant mean curvature in generalized Robertson-Walker spacetimes. Gen. Relativity Gravit. 27, 71-84 (1995)

4. Alías, L.J., Romero, A., Sánchez, M.: Spacelike hypersurfaces of constant mean curvature in spacetimes with symmetries. Publ. Real Soc. Mat. Esp. 1, 1-14 (2000)

5. Ahlfors, L.: Lectures on quasiconformal mappings. Van Nostrand, Toronto (1966)

6. Calabi, E.: Examples of Bernstein problems for some nonlinear equations. Proc. Symp. Pure Math. 15, 223-230 (1970)

7. Cheng, S.Y., Yau, S.T.: Maximal spacelike hypersurfaces in the Lorentz-Minkowski spaces. Ann. Math. 104, 407-419 (1976)

8. Duggan, K.L., Sharma, R.: Conformal Killing vector fields on spacetime solutions of Einstein's equations and initial data. Nonlinear Anal. 63, e447-e454 (2005)

9. Fernández, I., Mira, P.: Harmonic maps and constant mean curvature surfaces in $\mathbb{H}^{2} \times \mathbb{R}$, Am. J. Math. 129(4), 1145-1181 (2007)

10. Latorre, J.M., Romero, A.: New examples of Calabi-Bernstein problems for some nonlinear equations. Differential Geom. Appl. 15, 153-163 (2001)

11. Marsden, J.E., Tipler, F.J.: Maximal hypersurfaces and foliations of constant mean curvature in general relativity. Phys. Rep. 66, 109-139 (1980)

12. Wan, T.Y.: Constant mean curvature surface harmonic map and universal Teichmuller space. J. Differential Geom. 35, 643-657 (1992) 